\title{
Mapeamento com Software Coherent para Ablação de Flutter Atrial Atípico - Um Passo à Frente na Compreensão do Mecanismo da Arritmia
}

\author{
Coherent Map for Atypical Atrial Flutter - A Step Forward for the Understanding of the Arrhythmia Mechanism \\ Pedro A. Sousa, ${ }^{10}$ Sérgio Barra,,$^{2,3}$ Mariana Pereira, ${ }^{10}$ Luís Elvas $^{1}$ \\ Pacing \& Electrophysiology Unit, Cardiology Department, Centro Hospitalar e Universitário de Coimbra, ${ }^{1}$ Coimbra - Portugal \\ Hospital da Luz Arrábida V - Cardiology Department, ${ }^{2}$ Vila Nova de Gaia - Portugal \\ Royal Papworth Hospital NHS Foundation Trust - Cardiology Department, ${ }^{3}$ Cambridge - Reino Unido
}

\section{Apresentação do caso}

Uma mulher de 86 anos com antecedentes de fibrilação atrial paroxística foi encaminhada para ablação de flutter auricular (FLA) atípico. Ela nunca havia sido submetida a ablação por cateter antes. O ecocardiograma transtorácico revelou fração de ejeção do ventrículo esquerdo de $65 \%$, átrio esquerdo discretamente dilatado $(42 \mathrm{~mm})$ e regurgitação mitral moderada. O ECG de 12 derivações mostrou ondas $\mathrm{F}$ positivas em $\mathrm{V}$ 1 e nas derivações de membros inferiores, ondas $\mathrm{F}$ isoelétricas em DI e onda $\mathrm{F}$ negativa em aVL (Figura 1A). Dada a ativação concêntrica no seio coronário (SC), com uma duração do ciclo de taquicardia (TCL, tachycardia cycle length) de 330ms, (Figura 1B) um mapa de ativação atrial direito (AD), realizado com o cateter PentaRay ${ }^{\circledR}$, foi inicialmente analisado com a ferramenta $H D$ Coloring (CARTO ${ }^{\circ} 3 \mathrm{~V} 7$, Biosense Webster, CA, EUA). Foi revelada uma ativação precoce no septo interatrial com apenas $1 / 3$ da TCL (Figura 2). Um mapa de ativação do átrio esquerdo (AE) subsequente revelou uma grande área com cicatriz e provável bloqueio de condução em quase toda a parede anterior representada como uma linha branca de acordo com o recurso da ferramenta Extended Early Meets Late (EEML), e várias áreas com tempo de ativação local (LAT, local activation time) precoces - no apêndice atrial esquerdo (AAE), na face anterior da válvula mitral (VM) e no teto próximo ao segmento anterior da veia pulmonar superior direita (VPSD). Ele também mostrou duas zonas de reentrada, como estabelecido pelo recurso da ferramenta Early Meets Late (EML) (Figura 3A e S-3 A). Esses mapas sugeriam um provável circuito ao redor das veias pulmonares direitas, mas não explicavam a ativação da cor no AAE e na VM. Um novo algoritmo de mapeamento - Coherent (CARTO ${ }^{\circledR} 3 \mathrm{~V} 7$, Biosense Webster) revelou o circuito (Figura 3B e S-3 B). Qual é o mecanismo desse FLA atípico?

\section{Palavras-chave}

Flutter Atrial/etiologia; Condução; Arritmias Cardíacas; HD Coloring; Coherent Mapping; Técnicas Eletrofisiológicas Cardíaca/métodos.

Correspondência: Pedro A. Sousa •

Pacing \& Electrophysiology Unit, Cardiology Department, Centro

Hospitalar e Universitário de Coimbra, Coimbra, Portugal - Praceta Mota

Pinto Coimbra 3004 - Portugal

E-mail: peter senado2002@yahoo.com

Artigo recebī̄o em 08/12/2020, revisado em 20/04/2021, aceito em $12 / 05 / 2021$

DOI: https://doi.org/10.36660/abc.20201311

\section{Discussão}

Este caso destaca algumas características interessantes.

Primeiramente, tendo em consideração a ativação concêntrica no SC (Figura 1B), o mapeamento foi realizado inicialmente no AD com o software HD Coloring. O mapa bipolar revelou eletrogramas normais, definidos como voltagem acima de $0,3 \mathrm{mV}$, na maior parte da câmara (Figura S-2 A e S-2 B). Um mapa de ativação do AD de alta densidade revelou os pontos de LAT mais precoces no septo interatrial (Figura 2). A onda de propagação foi consistente com uma origem focal, mas como o sinal unipolar tinha uma deflexão inicial " $r$ " e apenas $1 / 3$ da TCL estava contido nessa câmara, um circuito exclusivamente no AD foi descartado (Vídeo suplementar 1).

Um mapa bipolar do AE foi subsequentemente realizado, revelando uma disfunção no $\mathrm{AE}$, com área de cicatriz densa e extensa na parede anterior, definida por uma voltagem abaixo de $0,1 \mathrm{mV}$, e algumas áreas de cicatriz irregular na parede posterior. (Figura S-2 A e S-2 B) O mapa de ativação de alta densidade realizado com o software $H D$ Coloring (Figura 3A e S-3 A), compreendendo toda a TCL, exibiu várias áreas de ativação precoce (no $\mathrm{AAE}$, a face anterior da VM e o teto próximo ao segmento anterior da VPSD). Duas áreas de reentrada, como definido pelo recurso da ferramenta $E M L$, também foram observadas - uma do AAE para a MV e outra no teto. Tanto a ferramenta EML quanto a EEML dependem do ciclo de taquicardia mapeado (que em nosso caso correspondeu à TCL), e são estabelecidos levando-se em consideração a diferença do LAT entre pontos adjacentes. ${ }^{1,2}$ Neste caso, uma vez que a diferença entre os pontos de LAT foi maior do que $25 \%$ da TCL ( $82 \mathrm{~ms}=0.25$ * 330ms), uma linha branca foi mostrada da VM à VPSD (poupando apenas uma pequena parte na parede anterior, próximo à VPSD) e também na parede posterior próximo à VPSD, sugerindo prováveis linhas de bloqueio de condução.

O mapa de propagação do AE (Vídeo Suplementar 2) sugeria um circuito ao redor das veias pulmonares direitas, com a onda de propagação movendo-se através da linha branca interrompida. No entanto, isso não explicava a ativação da cor no AAE e na VM. Mesmo depois de combinar os dois mapas (Figura S-1), várias questões permaneceram sem explicação:

1) Como poderia haver uma onda de propagação na parede anterior, dada a presença de extensa área de cicatriz?

2) Como ocorreu a ativação simultânea de diferentes áreas do $\mathrm{AE}$ ? 


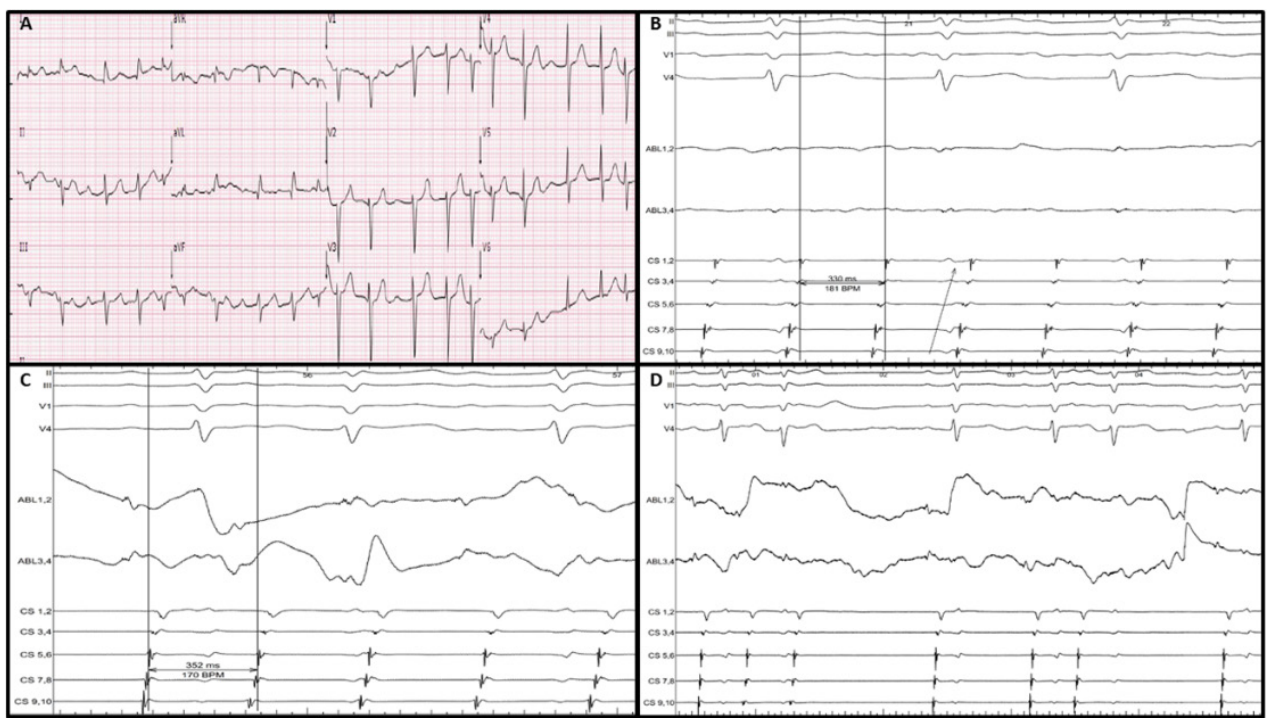

Figura 1 - Eletrocardiograma de doze derivações (A) e traçados intracardíacos obtidos durante o FLA (B) e durante as aplicações de radiofrequência (C e D).

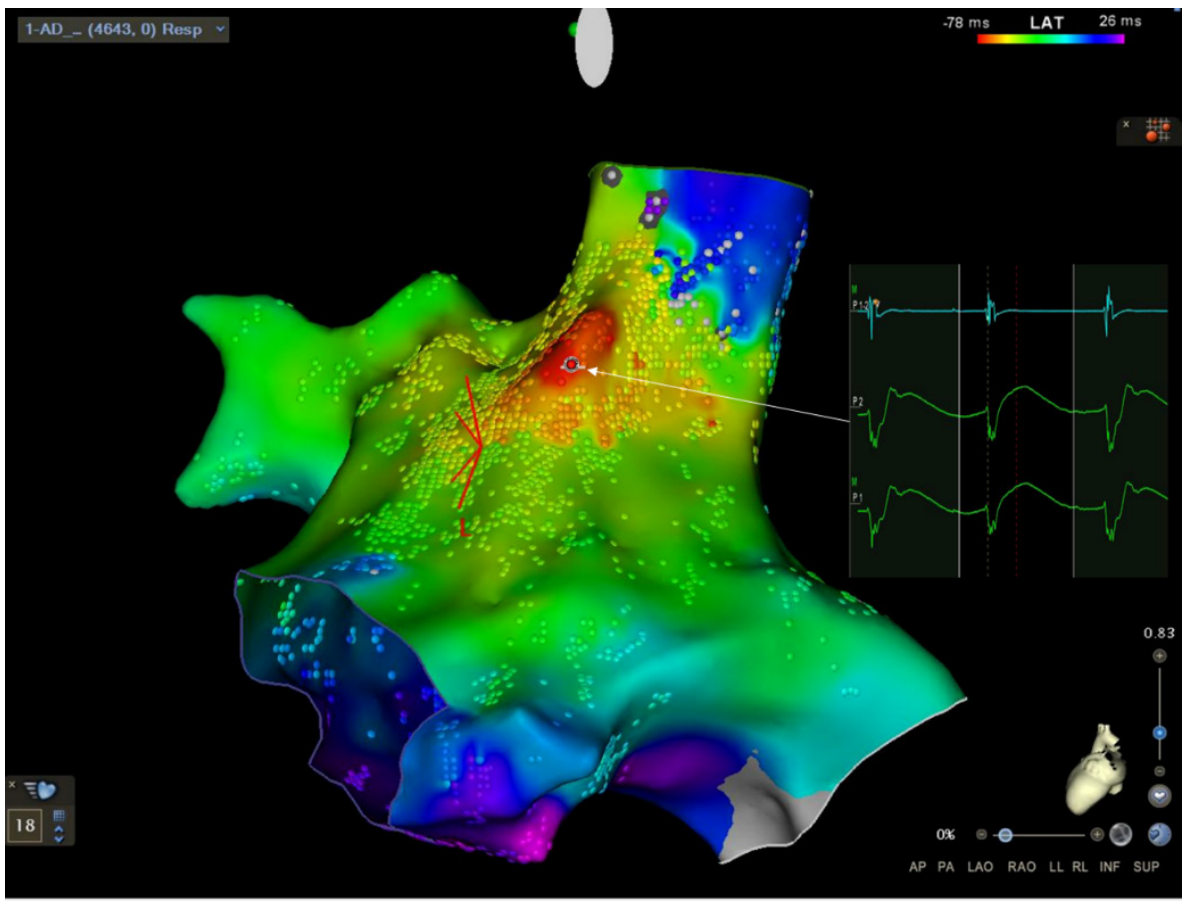

Figura 2 - Mapa de ativação de alta densidade do AD adquirida durante FLA com uma TCL de 330ms. 0 mapa foi realizado com o software HD Coloring, e incluiu 4.643 pontos, com 32\% da TCL e exibiu uma alta ativação septal centrifuga (vermelho indica as áreas com LAT mais precoce, enquanto laranja, amarelo, verde, azul e roxo indicam ativação progressivamente retardada) Os eletrogramas unipolares apresentaram uma deflexão inicial em " $r$ ". FLA: flutter atrial; AD: átrio direito; TCL: duração do ciclo de taquicardia. 


\section{Carta Científica}

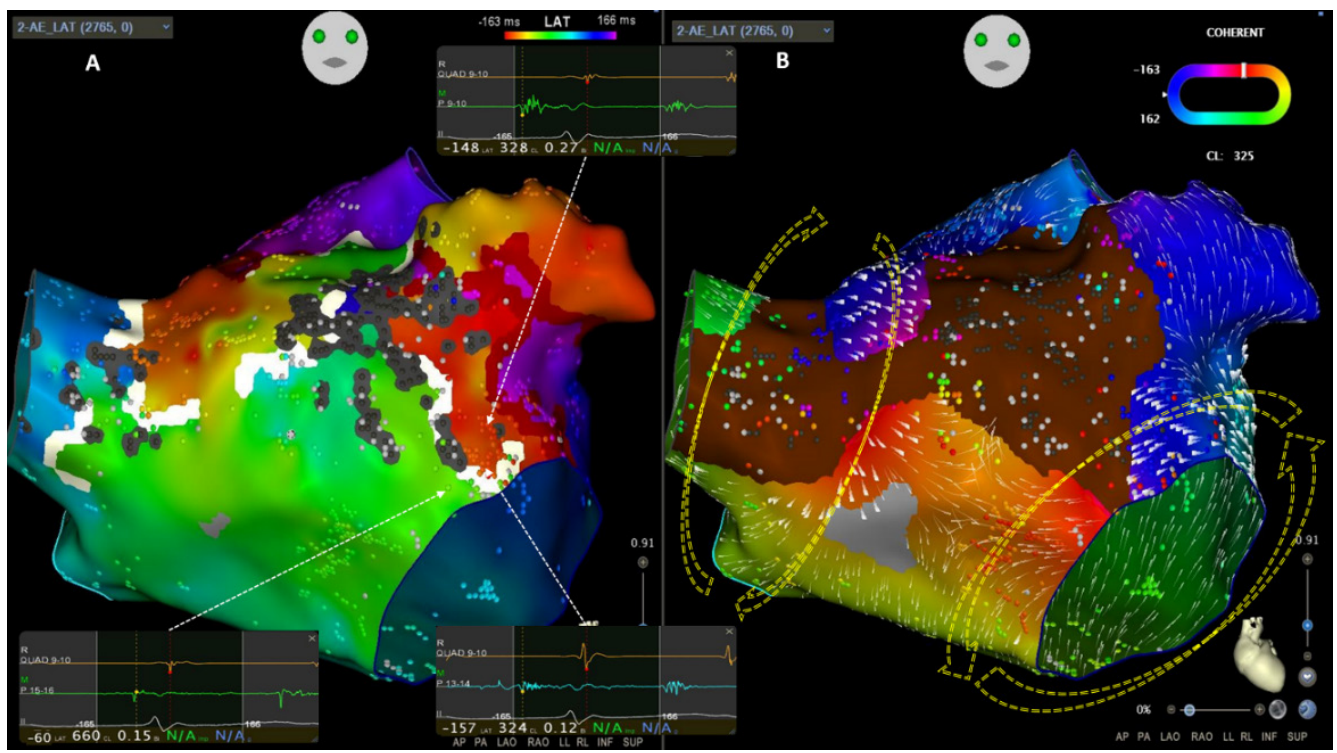

Figura 3 - Mapas de ativação atrial esquerda realizados com o cateter PentaRay ${ }^{\circledR}$, incluindo 2.765 pontos e $330 \mathrm{~ms}$ da duração do ciclo da taquicardia. O Mapa A foi realizado com o software HD Coloring (EML e EEML definidos em $75 \%$ e 25\%, respectivamente), com configurações de cicatriz bipolar em 0,03mV e um tamanho de área de cicatriz igual a 1 exibida como marcadores na cor cinza. 0 mapa de ativação revelou três áreas distintas de pontos $L A T$ iniciais - no teto, perto da VM anterior e no AAE - e duas zonas de provável reentrada, como definido por uma diferença entre pontos adjacentes superior a $75 \%$ da TCL (o Limiar EML) - no teto e entre o AAE e a VM, respectivamente. Havia uma provável linha de bloqueio de condução (exibida como uma linha branca) da VM até quase a VPSD, uma vez que a diferença entre os pontos LAT adjacentes em ambos os lados da linha eram maiores que $25 \%$ do ciclo de taquicardia mapeado. 0 mapa B foi realizado com o algoritmo de mapeamento Coherent, revelando uma zona SNO (condução lenta ou sem condução) (exibida em marrom) da VPSD até a face anterior da válvula mitral e também em direção ao AAE. Com o vetor de velocidade fixado em 17 (a velocidade lenta é representada por vetores mais grossos), a condução foi observada através da parede anterior próxima à VPSD - um circuito no sentido horário (seta amarela tracejada) ao redor das veias pulmonares direitas. 0 Coherent também revelou uma pequena área de condução lenta (eletrogramas fracionados como mostrado no Mapa A) perto da válvula mitral, sugestiva de um segundo circuito - uma onda de propagação no sentido anti-horário em torno da VM (seta amarela tracejada). EML: early meets late; EEML: extended early meets late; LAT: Tempo de ativação local; VM: válvula mitral; AAE: apêndice atrial esquerdo; TCL: duração do ciclo de taquicardia; VPSD: veia pulmonar superior direita.

3) As duas áreas de reentrada - no teto posterior e do AAE para a VM - correspondem a dois circuitos independentes ou somente a um circuito com condução passiva na outra área?

4) No caso de circuitos independentes, onde estão eles localizados e como pode a propagação ocorrer pela face anterior da VM se, de acordo com a ferramenta EEML, esta aparece bloqueada (como demonstrado pela linha branca ininterrupta)?

Como relatado anteriormente, ${ }^{2}$ no recurso da ferramenta HD Coloring, cada LAT requer uma voltagem de apenas $\geq 0,03 \mathrm{mV}$ para ser integrada ao mapa de ativação. Esta é a razão pela qual foi possível ver uma onda de ativação na parede anterior, apesar da presença de cicatriz extensa (voltagem do eletrograma bipolar abaixo de $0,1 \mathrm{mV}$ ). O entrainment poderia, em teoria, fornecer informações valiosas (como excluir um flutter atrial de 8 dígitos com um istmo comum), mas em nosso centro isso é normalmente realizado apenas se outros métodos não conseguirem explicar o circuito, devido ao pequeno risco de terminar a arritmia ou de sua degeneração em fibrilação atrial. ${ }^{3} \mathrm{O}$ mapeamento Ripple também pode ser útil nesses casos, pois supera algumas limitações dos mapas de LAT, como a anotação incorreta dos eletrogramas, e o fato de não ser influenciado pela janela de interesse. ${ }^{4}$
Recentemente, um novo algoritmo de mapeamento Coherent (CARTO ${ }^{\circledR} 3 \mathrm{~V} 7$, Biosense Webster) foi desenvolvido para abordar algumas das limitações associadas ao mapeamento de ativação atual. Resumidamente, o algoritmo de mapeamento Coherent leva em consideração o valor de LAT, o vetor de condução e a probabilidade de não-condutividade, e exibe o mecanismo de arritmia mais provável. ${ }^{3}$ Ele apresenta alguns novos recursos: 1) A presença dos vetores e sua direção e velocidade correspondentes e 2) Uma zona de "condução lenta ou sem condução" (SNO, slow or no conduction) exibida com uma cor marrom, representando uma área onde há condução lenta ou nenhuma condução.

A análise com este novo algoritmo de mapeamento (Figura 3B e S-3 B) permitiu-nos superar algumas das limitações do mapeamento de ativação convencional, mesmo com o recurso de HD Coloring: 1) A dificuldade em discriminar entre ativação ativa e passiva - em nosso caso, embora a janela de interesse tenha sido definida como igual à $\mathrm{TCL}$, a parede posterior e o $\mathrm{AAE}$ foram ativados com tal atraso que sua ativação continuou no próximo ciclo e, consequentemente, foram exibidos com uma cor vermelha, explicando porque eles pareciam estar simultaneamente ativados; 2) Cada LAT recebe uma 
anotação única, independentemente de seu fracionamento ou duração, o que pode confundir os operadores e até mesmo o próprio software. No presente caso, ao registrar os eletrogramas fracionados na válvula mitral lateral como LAT muito precoces, uma linha branca de bloqueio de condução foi exibida, o que dificultou a interpretação do mapa de ativação. Ao analisar as direções dos vetores e sua velocidade (velocidade lenta se representada por vetores mais grossos), observamos a condução através da parede anterior perto da VPSD - um circuito no sentido horário (seta amarela tracejada na Figura $3 \mathrm{~B}$ ) ao redor das veias pulmonares direitas. Entretanto, o mapeamento Coherent também revelou uma pequena área de condução lenta perto da válvula mitral, sugestiva de um segundo circuito - um flutter mitral no sentido anti-horário. Este circuito correspondia à área dos eletrogramas fracionados e poderia ter passado despercebido como uma linha branca, sugerindo que um bloqueio teria sido colocado pelo $H D$ Coloring (Figura 3A) devido ao motivo mencionado acima (cada LAT recebe um único registro, independentemente de seu fracionamento ou duração) (Figura 3B, S-3 B e Vídeo Suplementar 3).

Para confirmar nossa hipótese de um FLA com duplo loop, a etapa final foi a escolha do local para aplicação da energia de radiofrequência (RF). Inicialmente fechamos o gap perto da VPSD com alongamento imediato da TCL (Figura 1C). Após a entrega da energia de RF no aspecto anterior da válvula mitral, o FLA foi encerrado com sucesso (Figura 1D e Figura 4). O bloqueio bidirecional ao longo da linha de ablação foi confirmado com manobras de estimulação diferencial e com mapeamento de ativação repetido durante a estimulação do AAE (Vídeo Suplementar 4). Além disso, nenhuma arritmia adicional foi induzida subsequentemente. Após 5 meses de seguimento, o paciente permanece livre de qualquer arritmia sustentada.
Este caso destaca alguns dos novos recursos do algoritmo de mapeamento Coherent e sua utilidade, particularmente em pacientes com áreas de cicatriz extensa, eletrogramas fracionados e áreas de condução muito lenta. Ao exibir a de ativação mais lógica, o mapeamento Coherent superou as limitações relacionadas com a presença de condução muito lenta e o registro incorreto de eletrogramas fracionados, com a consequente exibição incorreta do bloqueio de condução, revelando um flutter atrial com duplo loop, e permitindo-nos tratar o paciente com sucesso.

\section{Contribuição dos autores}

Concepção e desenho da pesquisa e Análise e interpretação dos dados: Sousa PA; Obtenção de dados: Sousa PA, Pereira M; Redação do manuscrito: Sousa PA, Barra S; Revisão crítica do manuscrito quanto ao conteúdo intelectual importante: Barra S, Elvas L.

\section{Potencial conflito de interesse}

Não há conflito com o presente artigo

\section{Fontes de financiamento}

O presente estudo não teve fontes de financiamento externas.

\section{Vinculação acadêmica}

Não há vinculação deste estudo a programas de pósgraduação.

\section{Aprovação ética e consentimento informado}

Este artigo não contém estudos com humanos ou animais realizados por nenhum dos autores.

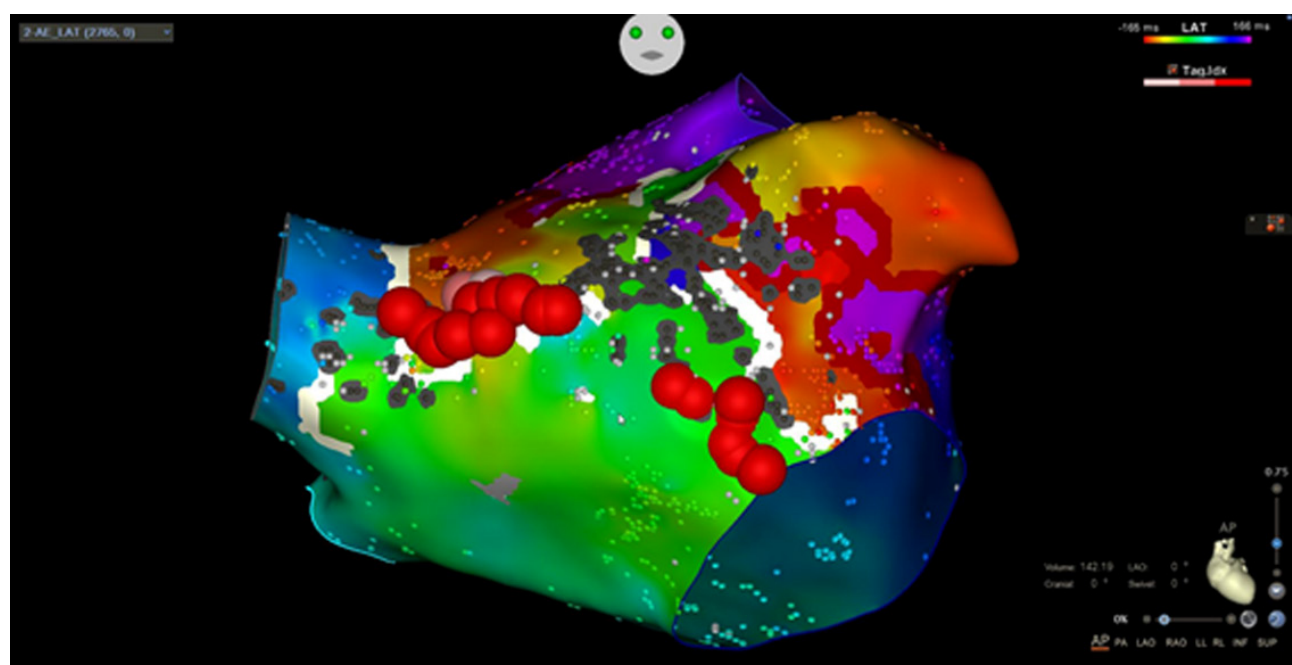

Figura 4 - Localização dos sitios de aplicação da radiofrequência, correspondendo aos locais de condução lenta, que permitiram a cessação do flutter atrial com duplo loop. 


\section{Carta Científica}

\section{Referências}

1. Sousa PA, Barra S, António N, Gonçalves L. HD Coloring for assessment of block along an ablation line. J Cardiovasc Electrophysiol. 2019;30(9):1692-3.

2. Sousa, PA, Barra S, Elvas L, Gonçalves L. HD Coloring for Atypical Flutter after Mitral Valve Repair: What's the mechanism? J Cardiovasc Electrophysiol 2020; 31(1): 252-5.
3. Anter E, Duytschaever M, Shen C,Strisciuglio T, Leshem E, Valdes FM, et al.. Activation Mapping With Integration of Vector and Velocity Information Improves the Ability to Identify the Mechanism and Location of ComplexScar-Related Atrial Tachycardias. Circ Arrhythm Electrophysiol. 2018;11(8): e006536.

4. Luther V, Cortez-Dias, N, Carpinteiro L, Sousa J, Balasubramaniam R, Agarwal S, et al. J Cardiovasc Electrophysiol. 2017;28(11):1285-94.

\section{* Material suplementar}

Para verificar as figuras, por favor, clique aqui.

Para assistir ao vídeo suplementar 1, por favor, clique aqui.

Para assistir ao vídeo suplementar 2, por favor, clique aqui.

Para assistir ao vídeo suplementar 3, por favor, clique aqui.

Para assistir ao vídeo suplementar 4, por favor, clique aqui. 